\title{
NEW POLYMORPHISMS IN REGULATORY REGIONS OF PORCINE $\mu$-CALPAIN GENE AND THEIR ASSOCIATION WITH CAPN1 TRANSCRIPT ABUNDANCE*
}

\author{
Katarzyna Ropka-Molik ${ }^{1 \star}$, Robert Eckert² ${ }^{2}$ Katarzyna Piórkowska ${ }^{1}$ \\ ${ }^{1}$ Laboratory of Genomics \\ ${ }^{2}$ Department of Animal Genetics and Breeding \\ National Research Institute of Animal Production, 32-083 Balice n. Kraków, Poland \\ •Corresponding author: katarzyna.ropka@izoo.krakow.pl
}

\begin{abstract}
The activity of calpains, in particular $\mu$-calpain, is associated with several processes occurring in muscle tissue postmortem and influences meat quality parameters. Therefore, the CAPN1 gene coding for large subunit of $\mu$-calpain is considered as a candidate gene associated with meat quality traits. The aim of our study was to identify new polymorphisms in regulatory regions of the porcine $C A P N 1$ gene and to estimate their impact on $C A P N 1$ transcript abundance. In the present study, 7 polymorphisms in the porcine $C A P N 1$ gene were identified, of which 5 were localized in introns (g.1195_1197insCCT; g.1429G>A; g.[4479A>G; 4526A>T; 4529_4530delAG]), one in 3' untranslated region $(\mathrm{g} .25676 \mathrm{C}>\mathrm{T})$ and one microsatellite sequence in promoter region (c.-155AGGG[3_5]). The analysed populations (a total of 451 gilts representing three pure breeds: Pietrain, Polish Landrace, Polish Large White and one conservation breed Pulawska) were not in Hardy-Weinberg equilibrium according to mutation g.25676C $>\mathrm{T}$ in 3' untranslated region (all breeds), g.1429G $>$ A (Pulawska pigs) and g.[4479A>G; 4526A>T; 4529_4530delAG] (PLW pigs). Furthermore, the analysed SNPs in the porcine CAPN1 gene were in linkage disequilibrium $(\mathrm{P} \leq \mathbf{0 . 0 5})$. The $C A P N 1$ transcript abundance was also estimated in two important muscles $(m$. longissimus dorsi, m. semimembranosus). In longissimus dorsi muscle, a significant effect of c.-155AGGG[3_5] polymorphism in promoter region on CAPN1 expression levels was determined. The c.-155AGGG[3/3] pigs showed a statistically higher $(\mathrm{P} \leq \mathbf{0 . 0 5})$ expression level of the $C A P N 1$ gene when compared to c.-155AGGG[4/4] homozygotes. The results obtained suggested that detected SNPs within regulatory regions of the $C A P N 1$ gene could be related to transcript level and activity of $\mu$-calpain. The selected polymorphisms are proposed to be associated with meat production traits.
\end{abstract}

Key words: calpain, $C A P N 1$ gene, polymorphism, expression level, pig

Calpains, which belong to the cysteine endopeptidase class, occur in all types of cells and their activity is subject to a suitable concentration of $\mathrm{Ca}^{2+}$ in the cell. The

*The study was supported by the Polish Ministry of Science and Higher Education (Project No. NN311 080640). 
presence and activity of the proteolytic enzymes calpains in muscle cells is related to the cell cytoskeleton proteolysis (Goll et al., 1998, 2003). This function is closely related to the changes in the muscles after slaughter, i.e. the process of meat maturation postmortem, tenderness and texture parameters. The high activity of calpains, in particular $\mu$-calpain, increases meat tenderness and softness (Kemp et al., 2010). Therefore, the CAPN1 gene coding for large subunit of $\mu$-calpain is considered as a candidate gene associated with meat quality traits.

In numerous domestic animals including pigs, research is conducted to find genetic basis of changes in the structure of calpain protein or differences in calpain activity. In the bovine $C A P N 1$ gene, Page et al. $(2002,2004)$ identified 38 polymorphisms localized in coding sequence and in introns and confirmed their association with important beef quality characteristics. Similarly, in the porcine CAPN1 gene several mutations in exons, introns and 3' untranslated (3'UTR) region were detected (Yang et al., 2007, 2008). The authors observed extremely significant differences in the distribution of genotypes among pig breeds being under strong selection for carcass meat content and wild boar. The results obtained could indicate that identified allele variants are related to lean meat percentage. Furthermore, Li et al. (2009), who estimated transcript abundance of the CAPN1 gene in muscle tissue, showed that decreased levels of calpain and calpastatin expression during development of neonatal pigs were associated with high protein accumulation and rapid muscle growth.

The use of the CAPN1 gene as a genetic marker in breeding programme could help to focus on improving pork quality while maintaining a satisfactory level of leanness. Therefore, the aim of our study was to identify new polymorphisms in regulatory regions of the porcine $C A P N 1$ gene and to estimate their impact on CAPN1 transcript abundance.

\section{Material and methods}

\section{Animals and tissues}

Analyses were performed on animals representing 4 pig breeds used in breeding programme as a dam-line (Polish Landrace - PL, Polish Large White - PLW), sire-line (Pietrain) and the genetic reserve breed of Puławska pigs. All animals were maintained in the Pig Performance Testing Station (SKURTCh) of the National Research Institute of Animal Production under the same housing and feeding conditions. All pigs (gilts with an average weight of $100 \mathrm{~kg}$ ) were fasted $48 \mathrm{~h}$ before the slaughter. Immediately after slaughter, blood samples were collected in tubes with EDTA and muscle tissues ( $m$. longissimus dorsi, $m$. semimembranosus) were collected and stored in RNAlater solution (Ambion Inc., Austin, USA). In total, the PCR-RFLP method was performed on 451 animals, while the Fragment Analysis application, which estimates the length of DNA fragment on capillary sequencer was carried out on 371 pigs. The evaluation of CAPN1 gene expression in two muscles was conducted on 180 gilts (PLW - 50, PL - 50, Puławska - 50, Pietrain - 30) for which also genotype frequencies were estimated. 


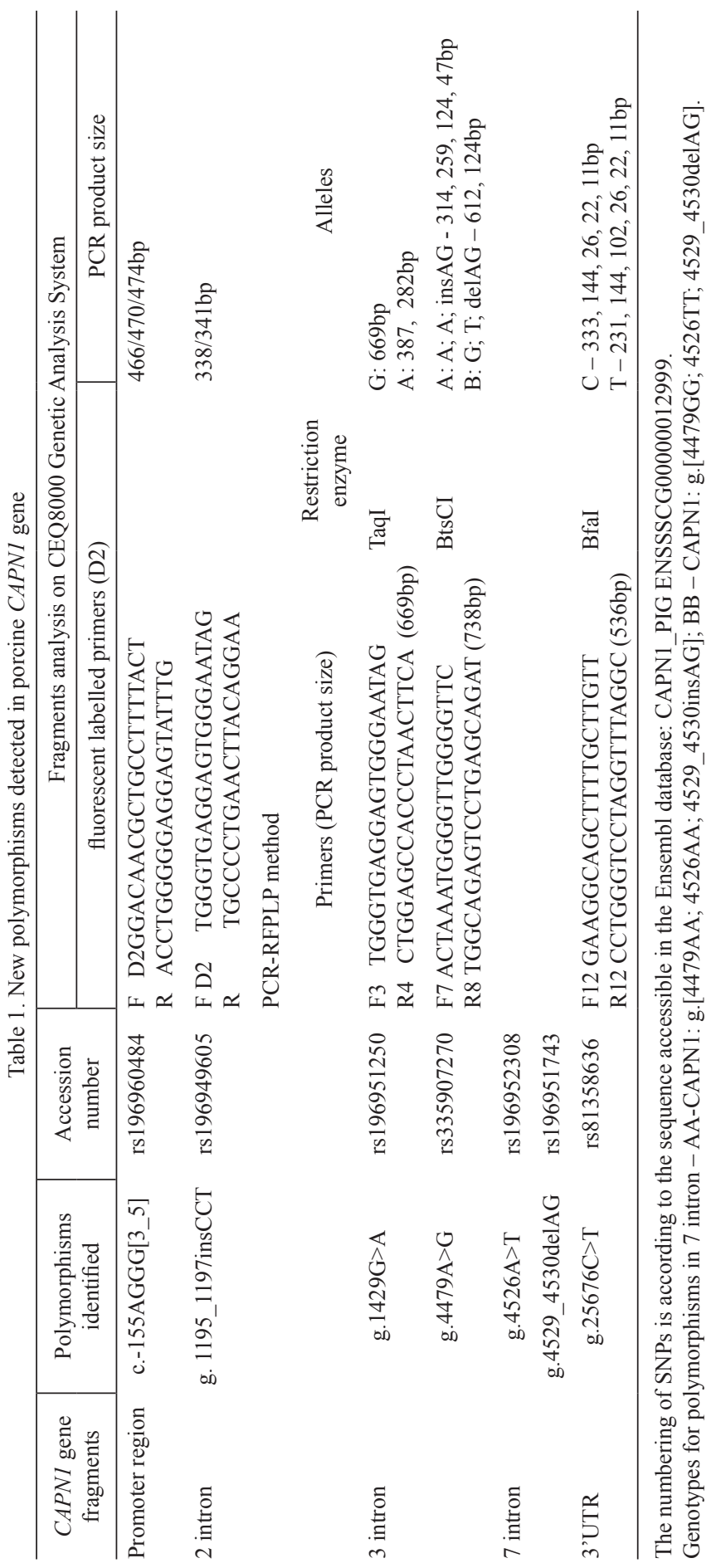




\section{Identification and genotyping of SNPs}

New polymorphisms in the CAPN1 gene were identified using the PCR-SSCP screening method. In the present study we analysed 9 fragments of $\mu$-calpain gene, including promoter region, 5' and 3' UTR, exons 3-8 and selected introns. The PCRSSCP analysis was performed on 96 gilts (24 sows of each breed) for each amplified fragment. The samples displaying the single strand conformation polymorphisms were sequenced with capillary sequencer CEQ8000 Genetic Analysis System using GenomeLab DTCS-Quick Start Kit (Beckman Coulter, Brea, CA, USA) according to the attached protocol.

To detect the individual mutations, the endonucleases were selected by using NEBCutter V2.0 (New England BioLabs, Frankfurt, Germany) (Table 1). After digestion, the PCR products were separated on 3\% and 5\% agarose gel. The detection of the c.-155AGGG[3_5] and g.1195_1197insCCT polymorphisms was performed using Fragment Analysis application, which estimates the length of DNA fragment, on sequencer CEQ8000 Genetic Analysis System with GenomeLab DNA Size Standard Kit - 600 (Beckman Coulter) (Table 1).

\section{Reverse transcription and quantification of $C A P N 1$ expression level}

The total RNA from $m$. longissimus dorsi and $m$. semimembranosus was isolated using TRI-Reagent (Sigma-Aldrich, Poznań, Poland) according to the method described by Chomczynski (1993). Evaluation of RNA quantity and quality was performed using the NanoDrop 2000 (Thermo Scientific, Wilmington, USA) and by $2 \%$ agarose gel electrophoresis. One $\mu \mathrm{g}$ of total RNA was transcribed to cDNA at $37^{\circ} \mathrm{C}$ using High Capacity cDNA Reverse Transcription Kit with random primers (Applied Biosystems), according to manufacturer's protocol.

The CAPN1 expression level was estimated with the use of two endogenous controls (OAZ1, RPL27) described previously by Piórkowska et al. (2011). Primers and probes for the porcine $C A P N 1$ gene were designed and synthesized by Applied Biosystems (Taqman gene expression assay ID: Ss03391458_m1, AY585336.1). The CAPN1 expression was evaluated on 7500 Real-Time PCR System using labelled TaqMan $^{\circledR}$ probes and TaqMan ${ }^{\circledR}$ Universal PCR Master Mix (Applied Biosystems). Reaction (in a total volume $25 \mu \mathrm{l}$ ) was performed in three repeats and the target gene was multiplexed with two endogenous controls (CAPN1 - FAM; OAZ1 - VIC; RPL27 - NED) according to the TaqMan Universal PCR Master Mix protocol: 2 initial steps at $50^{\circ} \mathrm{C}$ for 2 min (UNG incubation) and $95^{\circ} \mathrm{C}$ for $10 \mathrm{~min}$ (AmpliTaq Gold activation), and 40 cycles of $95^{\circ} \mathrm{C}$ for $15 \mathrm{sec}$ (denaturation) and $1 \mathrm{~min}$ at $60^{\circ} \mathrm{C}$ (annealing/extension). Reaction contained 12.5 $\mu 1$ TaqMan Universal PCR Master Mix, $0.5 \mu$ l of specific probes $R P L 27$ ( $250 \mathrm{nM}$ final concentration), $0.5 \mu 1$ of primers RPL27 (900 nM final concentration) and $1 \mu 1$ of assays (for CAPN1 and OAZ1), 2.5 $\mu \mathrm{l}$ of cDNA (1000 ng of total RNA for reverse transcription), water up to $25 \mu \mathrm{l}$. For all genes, the efficiency of real-time PCR reactions was defined by using the standard curve method. The standard curve points were prepared from subsequent serial dilutions $(1 ; 1 / 10 ; 1 / 100 / ; 1 / 1000$ of the RT product). The PCR efficiency was calculated as $\mathrm{E}=$ efficiency $\left(10^{[-1 / \mathrm{slope}]}\right)$, where the slope value was the directional factor of the curve. The relative mRNA abundance was calculated as $1 / \mathrm{E}^{(\mathrm{Ct})}(\mathrm{E}=$ efficiency; 
$\mathrm{Ct}=$ cycle) determined by the threshold applied to the maximum amplification of the standard curve. The normalization factor (NF) was calculated based on the geometric mean of the normalized quantity of the two endogenous genes. Relative quantity of CAPN1 mRNA abundance was calculated according to Pfaffl (2001).

\section{Statistical analysis}

The statistical analysis was performed using the GLM (SAS Institute, Cary, NC, v. 8.02, 2001) and the model used was:

$$
Y_{i j k l m}=\mu+g_{j}+h_{l}+e_{i j k l m}
$$

where:

$\mu$ - represents general mean,

$g_{j}$ - represents the effect of jth genotype,

$h_{l}$ - represents the effect of $1_{\text {th }}$ breed,

$e_{i j k l m}$ - represents the random error.

The Shapiro-Wilk test and the Levene's test were used to test if variables examined are normally distributed and to assess the equality of variances, respectively. The Hardy-Weinberg equilibrium was assessed by using Court Lab - HW calculator. The haplotype frequencies, D and D' values were estimated with an expectationmaximization algorithm as implemented in the Arlequin 3.11 and PowerMaker V3.0 software $(n=268)$. The bioinformatic analyses of likely transcription factor binding sites were performed using two prediction tools: TESS (Transcription Element Search System) and MathInspector (Genomatrix).

\section{Results}

\section{Identification of $C A P N 1$ polymorphisms}

In the present study, 7 polymorphisms in CAPN1 gene were identified, of which 5 were localized in introns, one in 3' untranslated region and one was microsatellite sequence in promoter region (Table 1). The g.[4479A $>$ G; 4526A $>$ T; 4529_4530delAG] mutations in intron 7 occurred jointly and were identified by using one restriction enzyme BtsCI. For all analysed polymorphisms three genotypes were determined, except for microsatellite sequence, where three alleles and six genotypes were identified. In all breeds, the most numerous genotype was c.-155AGGG[5/5] (43-85\%). In Puławska pigs, the heterozygotes had two-fold higher frequency compared to other breeds (c.-155AGGG[3/4] - 10\%; c.-155AGGG[3/5] - 28\%; c.-155AGGG[4/5] $15 \%)$.

The four pig breeds, which differ significantly in muscle mass and fat content, had also varied genotype frequencies for most investigated polymorphisms. In all breeds, the highest frequency of genotypes g.1429AA, BB - g.[4479GG; 4526TT; 4529_4530delAG] and g.1195_1197insCCT/insCCT were observed (about 67\%, 
$58 \%, 61 \%$, respectively). According to the g. $25676 \mathrm{C}>\mathrm{T}$ polymorphism, the most frequent were heterozygotes (71\%), while g.25676TT genotype was identified only in Pietrain and PL breeds. In Pietrain pigs, opposite distribution of g.25676C $>\mathrm{T}$ genotypes in comparison to other breeds was observed - the least frequent was g.25676CC (10\%) genotype and the most numerous was g.25676TT (30\%). Moreover, the Pietrain breed had the highest frequency of BB genotype - g.[4479GG; 4526TT; 4529_4530delAG] (81\%).

\section{Haplotype analysis}

The analysed polymorphisms in porcine $C A P N 1$ gene were in linkage disequilibrium $(\mathrm{P} \leq 0.05)$, the total number of possible haplotypes was nine, while three of them were the most frequent $(0.45,0.30,0.134$, respectively). The frequency of the rest of haplotypes was about $2 \%$ of investigated population (Table 2 ). The D (D') values for linkage between polymorphisms g.1429G $>$ A and g.1195_1197insCCT were 0.129 (0.95), between polymorphisms g.1429G $>$ A and g.[4479A $>$ G; 4526A $>$ T; 4529_4530delAG] were 0.095 (0.71). The linkage between g.[4479A $>$ G; 4526A>T; 4529_4530delAG] and g.25676C $>$ T polymorphisms was $0.067(0.56)$.

Table 2. Frequency of CAPN1 haplotypes according to Arlequin 3.11 and PowerMaker V3.0 software $(\mathrm{n}=268)$

\begin{tabular}{|c|c|c|c|c|c|}
\hline \multirow[b]{2}{*}{ No } & \multicolumn{3}{|c|}{ Haplotypes } & \multicolumn{2}{|c|}{ Frequencies } \\
\hline & g. 1195_1197insCCT & g. $1429 \mathrm{G}>\mathrm{A}$ & $\begin{array}{c}\text { g. }[4479 \mathrm{~A}>\mathrm{G} ; 4526 \mathrm{~A}>\mathrm{T} \\
\text { 4529_4530delAG] }\end{array}$ & g. $25676 \mathrm{C}>\mathrm{T}$ & \\
\hline 1. & insCCT & A & $\mathrm{B}$ & $\mathrm{T}$ & 0.45 \\
\hline 2. & insCCT & A & B & $\mathrm{C}$ & 0.30 \\
\hline 3. & delCCT & G & A & $\mathrm{C}$ & 0.134 \\
\hline 4. & insCCT & A & A & $\mathrm{C}$ & 0.029 \\
\hline 5. & delCCT & G & B & $\mathrm{C}$ & 0.025 \\
\hline 6. & delCCT & A & B & $\mathrm{C}$ & 0.022 \\
\hline 7. & delCCT & A & A & $\mathrm{C}$ & 0.022 \\
\hline 8. & delCCT & G & A & $\mathrm{T}$ & 0.013 \\
\hline 9. & insCCT & A & $\mathrm{A}$ & $\mathrm{T}$ & 0.005 \\
\hline
\end{tabular}

Alleles for polymorphisms in 7 intron A - CAPN1: g.[4479A; 4526A; 4529_4530insAG]; B - CAPN1: g.[4479G; 4526T; 4529_4530delAG].

\section{Association of identified polymorphisms and $C A P N 1$ expression level}

In longissimus dorsi muscle, the significant effect of c.-155AGGG[3_5] microsatellite in promoter region on transcript abundance was determined. The c.-155AGGG[3/3] pigs showed the statistically higher $(\mathrm{P} \leq 0.05)$ expression level of CAPN1 gene compared with homozygotes c.-155AGGG[4/4]. In semimembranosus muscle, similar expression levels in individual genotypes were identified, but without statistical significance (Figure 1). In both muscles only trends for other CAPN1 variants were observed. Homozygous genotypes of all investigated polymorphisms: AA - 
g.[4479AA; 4526AA; 4529_4530insAG], g.1429GG, g.25676CC, homozygotes without insertion - g.1195_1197insCCT were characterized by the lowest mRNA abundance, while heterozygotes were characterized by the highest expression (without statistical significance).

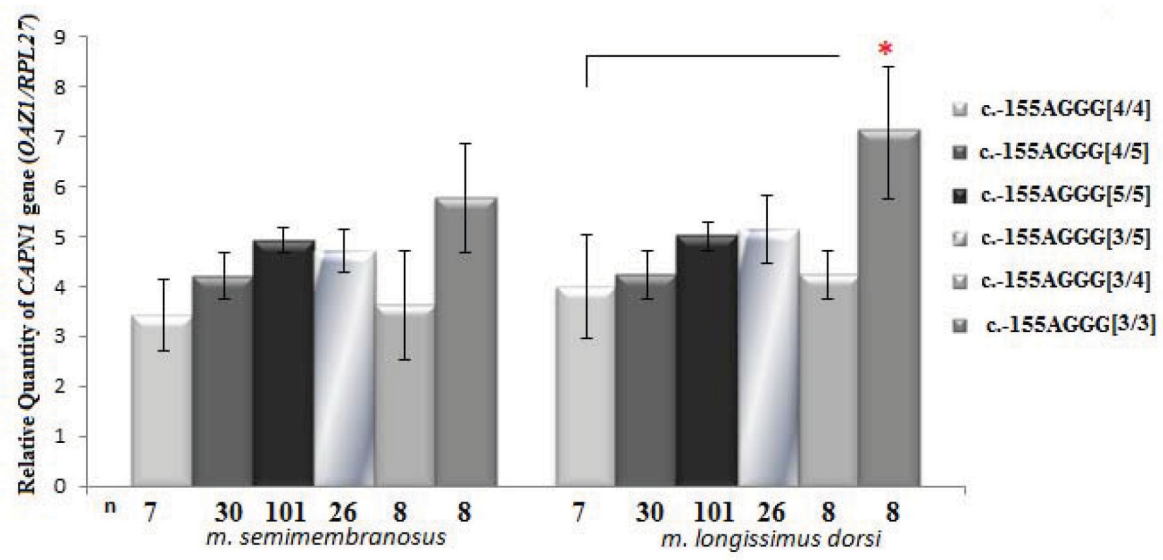

Figure 1. Relative Quantity (RQ) of the transcript abundance of different CAPN1: c.-155AGGG [3_5] genotypes $\left({ }^{*} \mathrm{P} \leq 0.05\right)$. The number of animals in each genotype group was presented under the figure. Data were presented as means \pm SEM

\section{Discussion}

Calpain 1 plays a critical role in the regulation of myogenesis or apoptotic process, cell mobility and cell signalling (Goll et al., 1998; Lee et al., 2000; Carragher and Frame, 2002; Norberg et al., 2008). Numerous studies have shown that mutations in the CAPN1 gene locus are associated with carcass meat content and meat quality parameters such as tenderness, water holding capacity, $\mathrm{pH}$ or meat colour (Oprządek et al., 2005; Cheong et al., 2008; Lee et al., 2008; Pinto et al., 2011). Until now, the knowledge about SNP polymorphisms in porcine CAPN1 gene and their association with productive traits and meat quality traits has been ambiguous and incomplete.

The genes encoding $\mu$-calpain have been studied in many meat breeds of domestic animals including chicken (Zhang et al., 2008), goat (Singh et al., 2012), and different breeds of cattle. In the bovine CAPN1 locus, Page et al. (2002) identified 38 SNPs, most of which were found in introns, and also two missense mutations in exons 9 and 13 (G316A and V530I) were determined. Furthermore, in Korean cattle, Cheong et al. (2008), who analysed 12 of 39 identified polymorphisms, showed that these variants of the $C A P N 1$ gene were in linkage disequilibrium $(\mathrm{P} \leq 0.05)$. The pairwise linkage disequilibrium analysis showed that the investigated polymorphisms can be assigned to two LD blocks spanning the $1.5 \mathrm{~kb}$ region from exon 5 to intron 7 and the $9 \mathrm{~kb}$ region from exon 11 to exon 22. The most numerous haplotypes had the frequency of 0.351 and 0.478 (Block 1 and 2, respectively). In our study, we also 
performed haplotype analysis which included 6 investigated polymorphisms (the microsatellite sequence was excluded from analysis due to low number of animals in genotype groups). The results indicated that CAPN1 gene is localized in the region of high linkage disequilibrium (LD).

The populations analysed were not in Hardy-Weinberg equilibrium according to mutation g.25676C $>\mathrm{T}$ in 3' untranslated region (all breeds), g.1429G $>$ A (Puławska pigs) and g.[4479A $>$ G; 4526A $>$ T; 4529_4530delAG] (PLW pigs). Probably, during selection frequencies of CAPN1 genotypes were changed, which might suggest a significant impact of the identified polymorphisms on the important pig traits. In Puławska pigs, which are a conservation breed and are not under a selection, the highest frequency of homozygotes without three nucleotide insertion g.1195_1197insCCT was observed. Furthermore, in this breed the distribution of c.-155AGGG[3_5] alleles was different and the largest number of heterozygotes in comparison to other breeds was identified. Similar results were obtained by Yang et al. (2008) who confirmed that the distribution of CAPN1 genotypes among Yorkshire, Min pigs and wild boar was highly significantly different $(\mathrm{P} \leq 0.05)$. The authors detected 11 SNPs of which 5 were localized in exons, 4 in introns and 2 in 3'UTR. According to Yang et al. (2008), preliminary analysis of association between polymorphisms in CAPN1 locus and lean meat percentage suggested the potential use of this gene as a genetic marker in breeding programme. In the porcine CAPN1 gene, Gandolfi et al. (2011) identified g.157T $>\mathrm{C}$ substitution in intron 5 and showed its effect on meat colour and myofibrillar particle size at $24 \mathrm{~h}$ postmortem. Additionally, CAPN1 g.157T > C genotype had no effect on calpain activity.

The polymorphisms identified in our study do not overlap with previously detected SNPs. The analysed mutations are located within regulatory sequences of CAPN1 gene and therefore, they might impact on $C A P N 1$ expression level. In the present research, the significant effect of c.-155AGGG[3_5] in promoter region on transcript abundance was determined. The c.-155AGGG[3/3] pigs showed a statistically higher $(\mathrm{P} \leq 0.05)$ expression level of $C A P N 1$ gene when compared to homozygotes c.-155AGGG[4/4]. On the other hand, in the analysed population the frequencies of some genotypes were low (especially for microsatellite polymorphism) and number of animals in each group was different; thus, the results obtained should be investigated further on a larger number of pigs. To date, the information available for changes in the level of CAPN1 gene expression in pigs and other farm animals is insufficient. The relationship between expression levels of $\mu$-calpain and rate of muscle growth was confirmed previously. Li et al. (2009) showed that calpain 1 and calpastatin transcript abundance was negatively correlated with protein accumulation in muscle tissue during neonatal period. The CAPN1 expression level corresponded to $\mu$-calpain protease activity and, as a result, was associated with growth rate and birth weight of piglets.

To confirm the association of novel polymorphisms in the CAPN1 gene and expression levels, the bioinformatic analysis of modification of transcription factor binding sites was performed (TESS, MathInspector) (Table 3). The analysis showed that c.-155AGGG[3_5] polymorphism changed complementarity of sequence binding three transcription factors - ZF07, GABF and EGFR, which may be related to 
differences in $C A P N 1$ expression levels between genotypes. Interesting results were obtained for three linkage polymorphisms in intron 7, which generated new binding sites, including for basic helix-loop-helix transcription factors - MyoD. The MyoD protein, belonging to the family of myogenic regulatory factors (MRFs), plays a key role in regulating myogenesis process via control of the proliferation and differentiation of muscle cells. Moreover, MyoD affects the activity of a number of proteins regulating muscle growth and development (Tapscott, 2005). Therefore, g.[4479A $\left.>\mathrm{G} ; 4526 \mathrm{~A}>\mathrm{T} ; 4529 \_4530 \mathrm{del} A \mathrm{~A}\right]$ polymorphisms in CAPN1 locus are considered to be a candidate SNP associated with meat production traits in pigs.

Table 3. Transcription factors whose binding sites in the DNA sequence have been modified by detected polymorphisms

\begin{tabular}{|c|c|c|c|c|}
\hline & Symbol & Transcription factor family name & $\begin{array}{c}\text { Compl. } \\
(\%)\end{array}$ & $\begin{array}{l}\text { Influence } \\
\text { of mutation }\end{array}$ \\
\hline & & & & c. -155 AGGG[3_5] \\
\hline \multirow[t]{4}{*}{$\begin{array}{l}\text { Promoter } \\
\text { region }\end{array}$} & ZF07 & $\mathrm{C} 2 \mathrm{H} 2$ zinc finger transcription factors 7 & 92 & $\begin{array}{l}+1(\text { c. }-155 \text { AGGG }[4]) \\
+2(\text { c. }-155 \mathrm{AGGG}[5])\end{array}$ \\
\hline & GABF & GA-boxes & 78 & $\begin{array}{l}+3(\text { c. }-155 \text { AGGG }[4]) \\
+5(\text { c. }-155 \text { AGGG }[5])\end{array}$ \\
\hline & EGFR & $\begin{array}{l}\text { EGR/nerve growth factor induced protein } \mathrm{C} \\
\text { and related factors }\end{array}$ & 88 & $\begin{array}{l}+1(\text { c. }-155 \text { AGGG }[4]) \\
+2(\text { c. }-155 \text { AGGG }[5])\end{array}$ \\
\hline & & & & g. 1195_1197insCCT \\
\hline \multirow[t]{2}{*}{2 intron } & IRFF & Interferon regulatory factors & 85 & - \\
\hline & PRDM & $\begin{array}{l}\text { PRDI-BF1 and RIZ homologous (PR) domain } \\
\text { proteins (PRDM) }\end{array}$ & 71 & - \\
\hline \multirow[t]{3}{*}{3 intron } & & & & g.1429G $>A$ \\
\hline & CLOX & CLOX and CLOX homology (CDP) factors & 94 & +2 \\
\hline & & g. $[4479 A>$ & ; $4526 \mathrm{~A}$ & T; 4529_4530delAG] \\
\hline \multirow[t]{6}{*}{7 intron } & APR1 & MAF and AP1 related factors & 82 & - \\
\hline & PRDM & $\begin{array}{l}\text { PRDI-BF1 and RIZ homologous (PR) domain } \\
\text { proteins (PRDM) }\end{array}$ & 71 & - \\
\hline & ETSF & Human and murine ETS1 factors & 86 & + \\
\hline & HAND & $\begin{array}{l}\text { Twist subfamily of class B bHLH transcription } \\
\text { factors }\end{array}$ & 87 & + \\
\hline & MyoD & Myoblast determining factors & 94 & + \\
\hline & RP58 & RP58 (ZFP238) zinc finger protein & 84 & + \\
\hline \multirow[t]{5}{*}{ 3'UTR } & & & & g. $25676 \mathrm{C}>\mathrm{T}$ \\
\hline & NF1F & Nuclear factor 1 & 92 & - \\
\hline & TALE & $\begin{array}{l}\text { TALE homeodomain class recognizing TG } \\
\text { motifs }\end{array}$ & 84 & - \\
\hline & $\mathrm{PBXC}$ & PBX1-MEIS1 complexes & 77 & - \\
\hline & CAAT & CCAAT binding factors & 83 & - \\
\hline
\end{tabular}

$(-)$ - loss of complementarity to binding sequences; $(+)$ - formation of a new binding site for transcription factors; $(+n)$ - formation of more than one new binding site for transcription factors; the new complementary sequences for transcription factors binding to CAPN1 promoter region were presented in comparison to c. -155 AGGG[3] allele. 
In summary, several novel SNPs within regulatory sequences of porcine CAPN1 gene were identified. The investigated polymorphisms were in linkage disequilibrium and according to g. $25676 \mathrm{C}>\mathrm{T}$ mutation populations analysed were not in Hardy-Weinberg equilibrium. The various distribution of CAPN1 genotypes between pig breeds differing in muscularity suggested that frequencies of CAPN1 genotypes might change during selection for improved meat parameters. The c.-155AGGG[3_5] microsatellite sequence localized in promoter region affected the transcript abundance of the $C A P N 1$ gene. Furthermore, the impact of g.[4479A $>\mathrm{G}$; 4526A $>$ T; 4529_4530delAG] polymorphisms on complementary sequences binding several transcription factors suggested that these mutations might be related to $C A P N 1$ expression level and $\mu$-calpain activity. The analysed polymorphisms are proposed to be associated with meat production traits, and thus further research in this area should be performed.

\section{References}

Carragher N.O., Frame M.C. (2002). Calpain: a role in cell transformation and migration. Int. J. Biochem. Cell. Biol., 34: 1539-1543.

Cheong H.S., Yoon D.H., Park B.L., Kim L.H., Bae J.S., Namgoong S., Lee H.W., $\mathrm{H}$ a n C.S., K i m J.O., C h e o n g I.C., S h in H.D. (2008). A single nucleotide polymorphism in CAPN1 associated with marbling score in Korean cattle. BMC Genetics, 9: 3.

Chomczynski P. (1993). A reagent for the single-step simultaneous isolation of RNA, DNA and proteins from cell and tissue samples. Biotechniques, 15: 532-537.

Gandolfi G., Pomponio L., Ertbjerg P., Karlsson A.H., Nanni Costa L., Lamets ch R., R u s s o V., D avoli R. (2011). Investigation on CAST, CAPN1 and CAPN3 porcine gene polymorphisms and expression in relation to post-mortem calpain activity in muscle and meat quality. Meat Sci., 88: 694-700.

Goll D.E., Thomp son V.F., Taylor R.G., O u ali A. (1998). The calpain system and skeletal muscle growth. Can. J. Anim. Sci., 78: 503-512.

Goll D.E., Th o m p s on V.F., Li H., We i W., C on g J. (2003). The calpain system. Physiol. Rev., 83: 731-801.

K e m p C.M., S e n sky P.L., B ard sle y R.G., B uttery P.J., P arr T. (2010). Tenderness - An enzymatic view. Meat Sci., 84: 248-256.

L e e M.S., K w on Y.T., L i M., P eng J., Frie d l and e r R.M., Ts a i L.H. (2000). Neurotoxicity induces cleavage of $\mathrm{p} 35$ to $\mathrm{p} 25$ by calpain. Nature, 405 : 360-364.

Le e H.L., S ante-Lhoutelli er V., Vigouroux S., Briand Y., Briad M., (2008). Role of calpains in postmortem proteolysis in chicken muscle. Poultry Sci., 87: 2126-2132.

L i Z., C a o B., Z ha o B., Yang X., F an M.Z., Yang J. (2009). Decreased expression of calpain and calpastatin mRNA during development is highly correlated with muscle protein accumulation in neonatal pigs. Comp. Biochem. Phys. A, 152: 498-503.

Norberg E., Gogvadze V., Otto M., Horn M., Uhlen P., Orrenius S., Zhivotovsk y B. (2008). An increase in intercellular $\mathrm{Ca} 2+$ is required for the activation of mitochondrial calpain to release AIF during cell death. Cell. Death Differ., 15: 1857-1864.

Oprządek J., Flisikowski K., Zwierzchowski L., Juszczuk-Kubiak E., Ros o ch acki S., D y m i ck i E. (2005). Associations between polymorphism of some candidate genes and growth rates, feed intake and utilisation, slaughter indicators and meat quality in cattle. Archiv. Tierzucht. Dummerstorf., 48: 81-87.

P a g e B.T., Cas as E., He a to n M.P., Culle n N.G., Hynd man D.L., Morris C.A., Craw ford A.M., Wh e le r T.L., K o o h mara i e M., K e ele J.W., S mith T.P.L. (2002). Evaluation of single-nucleotide polymorphisms in CAPN1 for association with meat tenderness in cattle. J. Anim. Sci., 80: 3077-3085. 
P a ge B.T., C a s a s E., Qua a s.L., Tha $11 \mathrm{~m}$ an R.M., Whe ele r T.L., Shackelford S.D., Koohmaraie M., White S.N., Bennett G.L., Keele J.W., Dikeman M.E., $\mathrm{S} \mathrm{m}$ ith T.P. (2004). Association of markers in the bovine CAPN1 gene with meat tenderness in large crossbred populations that sample influential industry sires. J. Anim. Sci., 82: 3474-3481.

Pfaff1 M.W. (2001). A new mathematical model for relative quantification in real-time RT-PCR. Nucleic Acids Res., 29: e45. doi: 10.1093/nar/29.9.e45.

Pinto L.F.B., Ferraz J.B.S., Pedrosa V.B., Eler J.P., Meirelles F.V., Bonin M.N., Rezende F.M., Carvalho M.E., Cucco D.C., Silva R.C.G. (2011). Single nucleotide polymorphisms in $C A P N$ and leptin genes associated with meat color and tenderness in Nellore cattle. Genet. Mol. Res., 10: 2057-2064.

Piórkowska K., Oczkowicz M., Różycki M., Ropka-Molik K., Piestrzynska- K a j t o c h A. (2011). Novel porcine housekeeping genes for real-time RT-PCR experiments normalization in adipose tissue: assessment of leptin mRNA quantity in different pig breeds. Meat Sci., 87: 191-195.

Singh L.V., Tripathi V., Sharma R., P andey A.K., Maitra A., Mishra B.P. (2012). Genetic polymorphism of CAPN1 gene in Sirohi goat. Intl. J. Meat. Sci., 2: 13-19.

Tap s c o t t S.J. (2005). The circuitry of a master switch: Myod and the regulation of skeletal muscle gene transcription. Development, 132: 2685-2695.

Yang X.Q., Li u H., G u o L.J., X u Y., Li u D. (2007). The mutation site analysis on CAPN1 gene of wild boar, Min pig and Yorkshire. Yi Chuan., 29: 581-586.

Yang X.Q., Li u H., Gu o L.J., Guan O.Z., X u Y., Liu D. (2008). Analysis of SNPs in partial exons and 3'UTR of CAPN1 in porcine. Yi Chuan, 30: 741-746.

Zh ang Z.R., Li u Y.P., J i ang X., D u H.R., Z h u Q. (2008). Study on association of single nucleotide polymorphism of CAPN1 gene with muscle fiber and carcass traits in quality chicken populations. J. Anim. Breed. Genet., 125: 258-264.

Received: 30 X 2013

Accepted: 18 II 2014 